\title{
Atitudes dos vizinhos de Serviços Residenciais Terapêuticos em relação aos pacientes psiquiátricos e aos serviços
}

\section{Neighbors attitudes toward psychiatric patients and residential services}

\section{Actitudes de los vecinos de Servicios Residenciales Terapéuticos hacia los pacientes psiquiátricos y los servicios}

\section{J oanna Gonçalves de Andrade Tostes*}

Universidade Federal de São João del-Rei - UFSJ, São João del-Rei, Minas Gerais, Brasil

\section{Marina Bandeira**}

Universidade Federal de São João del-Rei - UFSJ, São João del-Rei, Minas Gerais, Brasil

\section{Marcos Santos de Oliveira***}

Universidade Federal de São João del-Rei - UFSJ, São João del-Rei, Minas Gerais, Brasil

\begin{abstract}
RESUMO
O objetivo deste estudo foi avaliar as atitudes das pessoas que moram na vizinhança de Serviços Residenciais Terapêuticos (SRTs) em relação aos pacientes psiquiátricos e aos serviços de saúde mental, no município de Barbacena, Minas Gerais, Brasil. Foi avaliada uma amostra probabilística estratificada, composta por 110 vizinhos. Utilizou-se a Escala de Atitudes da Comunidade em Relação aos Doentes Mentais (CAMI-BR) e um questionário sociodemográfico e de informações sobre saúde mental. O resultado do escore global da CAMI-BR mostrou que, em geral, as atitudes dos vizinhos tenderam do neutro ao positivo. Os escores das subescalas foram significativamente diferentes entre si, com atitudes mais positivas na subescala de Benevolência e mais negativas na subescala de Autoritarismo. Observou-se uma ambivalência nas atitudes, apontando para a necessidade das pessoas receberem mais informações sobre os transtornos psiquiátricos e os serviços comunitários de saúde mental, visando a combater as atitudes estigmatizantes e a possibilitar uma melhor reinserção social dos pacientes na comunidade.
\end{abstract}

Palavras-chave: atitudes, pacientes, estigma, saúde mental.

\section{ABSTRACT}

The aim of this study was to evaluate the attitudes of people who live in the neighborhood of Therapeutic Residential Services (SRTs) toward psychiatric patients and mental health services, in the city of Barbacena, Minas Gerais, 
Brasil. A stratified random sample of 110 neighbors was evaluated using the Community Attitudes toward the Mentally Ill scale (CAMI-BR) and a questionnaire assessing sociodemographic and mental health informations. The result from the global score of CAMI-BR showed that, in general, neighbors attitudes ranged from neutral to positive. Subscales scores were significantly different from each other, with more positive attitudes in Benevolence subscale and more negative in the Authoritarianism one. The ambivalence observed in neighbors attitudes points to the need of providing more information about mental illness and community mental health services, in order to fight stigmatizing attitudes and to enable a better patient's social reinsertion in the community.

Keywords: attitudes, patients, stigma, mental health.

\section{RESUMEN}

El objetivo de este estudio fue evaluar las actitudes de las personas que viven en el vecindario de Servicios Residenciales Terapéuticos (SRTs) con relación a los pacientes psiquiátricos y los servicios de salud mental, en el municipio de Barbacena, Minas Gerais, Brasil. Fue evaluada una muestra aleatoria estratificada compuesta por 110 vecinos. Se utilizó la Escala de Actitudes de la Comunidad hacia los Enfermos Mentales (CAMI-BR) y un cuestionario sociodemográfico y de informaciones sobre salud mental. El resultado de la puntuación global de la CAMI-BR mostró que, en general, las actitudes de los vecinos tendían de neutral a positivo. Las puntuaciones de las subescalas fueron significativamente diferentes entre sí, con actitudes más positivas en la subescala de benevolencia y más negativas en la subescala de autoritarismo. Se observó una ambivalencia en las actitudes, indicando la necesidad de las personas de recibir más información sobre los trastornos psiquiátricos y los servicios comunitarios de salud mental, destinados a combatir las actitudes estigmatizantes y permitir una mejor reinserción social de los pacientes en la comunidad.

Palabras clave: actitudes, pacientes, estigma, salud mental.

\section{Introdução}

As atitudes das pessoas da comunidade em relação aos pacientes psiquiátricos são essenciais para a sua reinserção social (Organização Mundial de Saúde [OMS], 2001; Thornicroft \& Tansella, 2010). O bom acolhimento da população em geral e, particularmente, das pessoas que moram na vizinhança dos serviços de saúde mental é importante para um grande número de pacientes psiquiátricos, tendo em vista a alta prevalência destes transtornos e o grande impacto que geram (Abelha, Gonçalves, Legay, \& Lovisi, 2014; Zippay \& Lee, 2008). As estimativas indicam que, em conjunto, os transtornos psiquiátricos, neurológicos e decorrentes do uso de substâncias contribuem com $13 \%$ da Carga Global de Doenças no mundo (World Health Organization [WHO], 2013; OMS, 2008) e com 19\% no Brasil (Mari, 2014).

Dentre os impactos negativos dos transtornos psiquiátricos sobre os pacientes destacam-se o sofrimento, a baixa qualidade de vida, a falta de integração social e a perda da capacidade de trabalho e 
autonomia (Thornicroft \& Tansella, 2010; Bandeira \& Barroso, 2005). Frequentemente, estes prejuízos encontram-se associados a atitudes negativas da sociedade em relação a esses pacientes, prejudicando a sua reinserção social (Thornicroft \& Tansella, 2010; OMS, 2008; Abelha et al., 2014; Gonçalves, Abelha, Legay, \& Lovisi, 2008). Essas atitudes negativas estão relacionadas ao conceito de estigma. A percepção de que um indivíduo é socialmente inaceitável, por apresentar características discrepantes dos demais, sendo por isso desvalorizado, é conceituada como estigma público (Corrigan \& Watson, 2002). Apesar das acentuadas diferenças socioeconômicas e culturais entre as populações avaliadas em alguns países, ainda assim foram encontrados níveis de estigma igualmente elevados em relação a esses pacientes (Mirnezami, Jacobsson, \& Edin-Liljegren, 2015; Liu, et al., 2015; Barke, Nyarko, \& Klecha, 2011; Griffiths et al., 2006; Maclean, 1969).

O impacto das atitudes das pessoas da comunidade em relação aos pacientes psiquiátricos tornou-se mais evidente após a desinstitucionalização psiquiátrica, na qual a prestação de cuidados passou a ser realizada em serviços comunitários de saúde mental (Morgado \& Lima, 1994; Bandeira \& Barroso, 2005). No entanto, para que esse processo possa ser efetivo, é necessária a expansão e a consolidação de uma rede de assistência aos pacientes psiquiátricos, que atenda às necessidades específicas do território onde se localiza. Não pode resultar, portanto, na mera desospitalização desses pacientes e em cuidados insuficientes (Amorim \& Dimenstein, 2009a).

Dentre os serviços comunitários de saúde mental criados em substituição aos hospitais psiquiátricos, destacam-se os Serviços Residenciais Terapêuticos (SRTs), que incluem em seus objetivos a progressiva reinserção dos pacientes na comunidade (Ministério da Saúde, 2004, 2011). Os SRTs são moradias no meio urbano para atender pacientes egressos de longas internações em hospitais psiquiátricos ou em hospitais de custódia (Ministério da Saúde, 2011, 2015). Configuram-se como um tipo de serviço que possibilita a convivência urbana dos pacientes com transtornos psiquiátricos, anteriormente marcada pelo isolamento e restrita ao ambiente dos hospitais (Amorim \& Dimenstein, 2009a).

Um dos principais requisitos para o sucesso da implantação destas modalidades de serviços comunitários é o estabelecimento de boas relações entre os pacientes e os residentes locais. No entanto, a vizinhança desses serviços frequentemente apresenta reservas em relação aos pacientes (Thornicroft \& Tansella, 2010). A fim de potencializar o estabelecimento de boas redes de suporte social e de cuidados em saúde, deve ser viabilizada uma maior articulação entre a gestão, os serviços, a comunidade e outros setores, tais como o de educação e o de assistência social. Há um espaço entre a indiferença 
e a solidariedade dos residentes locais em relação aos pacientes que pode ser adequadamente trabalhado (Amorim \& Dimenstein, 2009b). A importância das reações da comunidade é destacada em um documento da OMS (2001), que descreve dez recomendações para o cuidado aos pacientes. Uma delas é a de promover a educação do público, com campanhas visando ao conhecimento e à sensibilização das pessoas em relação aos transtornos psiquiátricos. Essas ações têm como finalidade reduzir o estigma e estimular a aproximação entre a comunidade, os pacientes e os familiares. Segundo Donabedian (1990), a aceitabilidade de um serviço pela comunidade é uma das qualidades de um serviço de saúde mental. O Ministério da Saúde (2010) reconhece que o estigma se constituiu como um dos entraves para a reinserção social dos pacientes psiquiátricos e que são necessárias políticas públicas intersetoriais para combatê-lo.

Para identificar estudos que tenham avaliado as atitudes da população em relação aos pacientes psiquiátricos, em particular da vizinhança próxima às suas moradias, foi realizada uma busca nos indexadores SciELO, Medline, Lilacs e Psycl NFO As combinações das seguintes palavras-chave, em português e inglês, foram utilizadas: "estigma público, paciente psiquiátrico, transtorno mental, atitudes da comunidade, vizinhos, vizinhança, residências terapêuticas, discriminação, public stigma, psychiatric patient, mentally ill, community attitudes, neighbors, neighborhood, therapeutic residences, residential care, discrimination". No contexto internacional, foram encontrados 10 estudos quantitativos que avaliaram pessoas da vizinhança de serviços residenciais para pacientes psiquiátricos. Foi identificada uma única pesquisa nacional com o objetivo de validar uma escala de atitudes em relação aos pacientes psiquiátricos (CAMI-BR), cuja população-alvo foi composta por vizinhos de SRTs (Abelha, Siqueira, Legay, Yang, Valencia, Sarução, \& Lovisi, 2015).

Dentre os 10 estudos internacionais, seis encontraram atitudes positivas das pessoas em relação aos pacientes psiquiátricos e sua presença na vizinhança (Zippay \& Lee, 2008; Aubry, Tefft, \& Currie, 1995; Arens, 1993; Rabkin, Muhlin, \& Cohen, 1984; Cook, 1997; Wahl, 1993). Três pesquisas encontraram atitudes negativas dos vizinhos (Reda, 1995; Trute \& Loewen, 1978; Wolff, Pathare, Craig, \& Leff, 1996). Em um estudo, o autor concluiu que, de forma geral, não houve alteração das atitudes das pessoas em relação aos pacientes, antes e após a instalação do serviço na vizinhança, mas em um dos aspectos avaliados, observou-se que as atitudes se tornaram mais negativas (Brunton, 1997). Com relação ao único estudo nacional encontrado, os resultados indicaram atitudes que tenderam do neutro ao positivo (Abelha et al., 2015). Considerando a contradição dos resultados dos estudos internacionais e a carência de estudos nacionais nesta temática, a presente pesquisa teve como objetivo 
avaliar as atitudes das pessoas que moram na vizinhança de SRTs em relação aos pacientes psiquiátricos e aos serviços. Este estudo pode ser classificado como uma pesquisa avaliativa do tipo somativa (Selltiz, Wrightsman, \& Cook, 1987; Smith, 1997), pois permite analisar o impacto de um tipo de serviço de saúde mental sobre as pessoas da comunidade, como um dos indicadores de seus resultados.

\section{Método}

\subsection{Participantes}

A população-alvo foi composta por habitantes do município de Barbacena, em Minas Gerais, que preencheram os seguintes critérios de inclusão: idade igual ou superior a 18 anos, ambos os sexos, morar nas ruas de Serviços Residenciais Terapêuticos (SRTs) instalados no endereço há, pelo menos, um ano. Os moradores de 22 ruas, onde estavam localizados 24 SRTs, foram elegíveis para participar do estudo. Em cada uma dessas ruas, foram consideradas as 20 casas mais próximas, situadas ao lado e em frente de cada um dos SRTs, a partir das quais foi selecionada a amostra. A delimitação da proximidade da população-alvo em relação aos SRTs, deste estudo, se assemelhou à realizada por Arens (1993) e Cook (1997). Mediante a presença de obstáculos, tais como estabelecimentos comerciais, terrenos baldios, igrejas e condomínios fechados, foram consideradas as casas imediatamente seguintes, como no estudo nacional de Abelha et al. (2015). Embora tenha sido prevista a exclusão de pessoas com dificuldades de compreensão das questões dos instrumentos de medida, avaliadas por meio de uma Técnica de Sondagem (Guillemin, Bombardier, \& Beaton, 1993), apenas um sujeito foi excluído por essa razão.

Foi selecionada uma amostra probabilística estratificada, na qual os estratos foram as ruas dos SRTs. Em cada um desses estratos, foi realizada uma seleção aleatória simples de casas, com a mesma probabilidade de serem selecionadas para compor a amostra. Esse método permite obter uma amostra representativa da populaçãoalvo, pois são selecionados elementos de todos os estratos (Selltiz et al., 1987; Contandriopoulos, Champagne, Potvin, Denis, \& Boyle. 1994). Essa característica possibilita a generalização estatística dos resultados encontrados na amostra para a população-alvo da qual ela foi retirada, aumentando a validade externa do estudo. Em cada casa selecionada, foi entrevistado um dos moradores que aceitou participar do estudo e que preencheu os critérios de inclusão e exclusão. O tamanho da amostra foi determinado por métodos estatísticos, baseado nas orientações de Snedecor e Cochran (1971) 
e Resende, Sturion e Pereira (2004), considerando os objetivos do presente estudo. A amostra final foi composta por 110 sujeitos, sendo cinco sujeitos em cada uma das 22 ruas, o que garantiu um poder de amostra mínimo igual a 95\%. Para fazer a seleção probabilística da amostra, foram gerados previamente números aleatórios distintos de 1 a 20 para cada rua, correspondentes às 20 casas mais próximas de cada um dos SRTs. Os primeiros cinco números aleatórios gerados para cada rua corresponderam às casas selecionadas para compor a amostra.

\subsection{Instrumentos}

Escala de Atitudes da Comunidade em Relação aos Doentes Mentais (CAMI-BR): esta escala foi elaborada por Taylor e Dear (1981), adaptada para o contexto brasileiro por Gonçalves et al. (2008) e validada por Abelha et al. (2015). É composta por 40 itens, divididos em quatro subescalas com 10 itens cada, que avaliam as atitudes das pessoas da comunidade em relação aos pacientes psiquiátricos e aos serviços de saúde mental, nos domínios: 1) Autoritarismo (hospitalização e tratamento coercivo); 2) Restrição Social (distância social, concepções de normalidade e periculosidade); 3) Benevolência (envolvimento e responsabilidade da sociedade) e 4) Ideologia Comunitária de Saúde Mental (impacto dos serviços e aceitação dos princípios da desinstitucionalização). As alternativas de respostas aos itens estão distribuídas em uma escala do tipo Likert de cinco pontos, variando de 0 (Concordo totalmente) a 4 (Discordo totalmente). Como a escala possui uma variação da ordem da conotação positiva e negativa dos itens, é necessária a recodificação de alguns deles. Realizada a recodificação, o escore global é obtido por meio do somatório das respostas aos itens, indicando que quanto maior o escore, mais positivas são as atitudes (Abelha et al., 2015). A escala apresenta indicadores de qualidades psicométricas adequados, em termos de fidedignidade (Abelha et al., 2014) e de validade (Abelha et al., 2015).

Questionário sociodemográfico e de informações sobre saúde mental: este questionário avaliou as características sociodemográficas dos entrevistados, tais como sexo, estado civil, escolaridade e religião. Incluiu também questões referentes ao contato e informações que eles possuíam sobre transtornos psiquiátricos e sobre os serviços de saúde mental. Essas questões foram elaboradas com base na literatura e submetidas, juntamente com a escala CAMI-BR, a um estudo-piloto com 13 sujeitos (aproximadamente 10\% da amostra), de acordo com as recomendações de Canhota (2008). Foi constatada a facilidade de compreensão de ambos os instrumentos. 


\subsection{Procedimento de coleta de dados}

Este estudo foi aprovado pela Comissão de Ética em Pesquisa envolvendo Seres Humanos (CEPES) da Universidade Federal de São João del-Rei, em Minas Gerais, e pela Coordenação dos Serviços Residenciais Terapêuticos do município de Barbacena. As entrevistas foram conduzidas de acordo com os parâmetros éticos, tendo sido assegurado o sigilo e a confidencialidade das informações fornecidas. Os sujeitos receberam informações sobre os objetivos e procedimentos do estudo. Sua participação foi voluntária, mediante a leitura e a assinatura de um Termo de Consentimento Livre e Esclarecido.

Os entrevistadores foram treinados previamente para realizar a aplicação dos instrumentos de medida, a fim de garantir a qualidade e a padronização da coleta de dados. Em geral, os sujeitos foram convidados a participar do estudo pessoalmente, em suas respectivas casas. Em alguns casos, o contato via telefone foi feito quando não havia sido encontrado nenhum morador na casa e havia um número disponível no catálogo telefônico. Foram entrevistados, imediatamente, aqueles que concordaram em participar e apresentaram disponibilidade. Houve o agendamento das entrevistas, nos casos em que os sujeitos aceitaram participar do estudo, mas não estavam disponíveis naquele momento.

\subsection{Análise de dados}

A análise dos dados foi realizada por meio do software Statistical Package for the Social Sciences (SPSS), na versão 20.0. Por meio do teste de Kolmogorov-Smirnov, foi constatada a normalidade $(p>$ $0,05)$ dos dados do escore global e de três subescalas da CAMI-BR (escore global, $\mathrm{K}-\mathrm{S}=0,91, \mathrm{p}=0,38$; Sub. $1, \mathrm{~K}-\mathrm{S}=0,75, \mathrm{p}=0,63$; Sub. $2, \mathrm{~K}-\mathrm{S}=1,22, \mathrm{p}=0,10$; Sub. $3, \mathrm{~K}-\mathrm{S}=0,90, \mathrm{p}=0,39$ ). Para a quarta subescala, foi confirmada a normalidade dos dados por meio dos coeficientes de Assimetria (Skewness) e de Curtose (Kurtosis), também indicadores da distribuição normal dos dados quando seus coeficientes estão situados na faixa entre -2 e 2 (George \& Mallery, 2010) (Assimetria $=-1,02$; Curtose $=1,71$; DP $=0,54$ ).

Para descrever a amostra e os escores da escala CAMI-BR, foi utilizada a estatística descritiva, com cálculo de médias, desviospadrão e porcentagens. Foi realizada a análise de variância (ANOVA) de medidas repetidas e o teste post hoc de Bonferroni, para verificar se havia diferenças estatisticamente significativas entre as médias das quatro subescalas da CAMI-BR e identificar em quais domínios as atitudes das pessoas eram mais positivas ou mais negativas. Foi realizada, ainda, a análise de variância de postos, com o teste de 
Friedman, para identificar qual item de cada uma das quatro subescalas da CAMI-BR foi mais relevante dentre os demais itens.

\section{Resultados}

\subsection{Descrição da amostra}

A idade média dos sujeitos foi de 47,39 anos, variando de 19 a 81 anos. A maioria era do sexo feminino $(74,5 \%)$, casada ou vivia com um companheiro $(60 \%)$, possuía filhos $(75,5 \%)$, em geral adultos $(48,2 \%)$ e que residiam na mesma casa $(69,9 \%)$. A média de anos de escolaridade foi de 11,11 anos, variando de dois a 22 anos, sendo que $33,6 \%$ possuíam o ensino médio completo. A maioria residia em imóvel próprio $(84,5 \%)$ e praticava alguma religião $(94,5 \%)$, principalmente a Católica $(79,8 \%)$. Dentre os que trabalhavam $(47,3 \%)$, a principal ocupação era o trabalho diário remunerado $(36,4 \%)$.

Pouco mais da metade dos sujeitos tinha algum familiar com transtorno psiquiátrico $(52,7 \%)$ e já tinha recebido informações sobre esses transtornos $(53,6 \%)$. A maioria já teve contato com algum paciente psiquiátrico $(96,4 \%)$, acreditava que eles não são culpados por sua própria condição $(99,1 \%)$ e que não conseguiriam controlar seus comportamentos $(85,5 \%)$. Dos entrevistados, $42,7 \%$ já procuraram um psicólogo ou psiquiatra para avaliação de transtorno psiquiátrico, $20 \%$ já haviam utilizado um serviço de saúde mental e $55,5 \%$ tinham algum familiar que já fez uso desses serviços. A maioria conhecia alguma residência terapêutica $(92,7 \%)$ e sabia que havia uma delas próxima de sua casa $(82,7 \%)$, mas apenas a minoria foi informada sobre sua instalação $(11,8 \%)$.

\subsection{Análise do escore global e por subescalas da CAMI-BR}

$O$ resultado do escore global $(M=2,90 ; D P=0,36)$ indicou que as pessoas que moravam na vizinhança dos SRTs possuíam, em média, atitudes que tendiam do neutro (escore 2 ) ao positivo (escores de 3 a 4). Com relação às subescalas, os resultados foram semelhantes, variando do neutro ao positivo para as subescalas de Autoritarismo $(M=2,38 ; D P=0,43)$ e de Restrição Social $(M=2,79 ; D P=0,50)$. Nas outras duas subescalas, as atitudes se mostraram positivas: Benevolência $(M=3,31 ; D P=0,40)$ e Ideologia Comunitária de Saúde Mental ( $M=3,13 ; D P=0,54)$.

Os resultados das comparações entre as subescalas, por meio da análise de variância ANOVA de medidas repetidas, indicaram diferenças significativas $(F=153,61 ; p<0,001)$. $O$ teste post hoc de Bonferroni mostrou que todas as subescalas eram significativamente 
diferentes entre si, tendo em vista que nenhum dos intervalos de confiança (IC95\%) continha o valor zero. As atitudes dos sujeitos se mostraram mais positivas na subescala de Benevolência (envolvimento e responsabilidade da sociedade), quando comparadas às subescalas de Ideologia Comunitária de Saúde Mental, de Restrição Social e de Autoritarismo. Por outro lado, atitudes mais negativas foram observadas na subescala de Autoritarismo (hospitalização e tratamento coercivo), quando comparada à de Restrição Social, de Benevolência e de Ideologia Comunitária de Saúde Mental (Tabela 1).

\begin{tabular}{|c|c|c|c|}
\hline \multicolumn{4}{|c|}{$\begin{array}{l}\text { Análise comparativa dos escores nas subescalas da CAMI-BR por meio da ANOVA de medidas } \\
\text { repetidas e teste post hoc de Bonferroni }\end{array}$} \\
\hline Variáveis & $\begin{array}{l}\text { Médias } \\
\text { (DP) }\end{array}$ & IC95\%* & $p$ \\
\hline Subescala 1 da CAMI-BR - Autoritarismo & $2,38(0,43)$ & \multirow{2}{*}{$(-0,53 ;-0,29)$} & \multirow{2}{*}{$0,000^{* *}$} \\
\hline Subescala 2 da CAMI-BR - Restrição Social & $2,79(0,50)$ & & \\
\hline Subescala 1 da CAMI-BR - Autoritarismo & $2,38(0,43)$ & \multirow{2}{*}{$(-1,06 ;-0,81)$} & \multirow{2}{*}{$2,000^{* *}$} \\
\hline Subescala 3 da CAMI-BR - Benevolência & $3,31(0,40)$ & & \\
\hline Subescala 1 da CAMI-BR - Autoritarismo & $2,38(0,43)$ & \multirow{2}{*}{$(-0,90 ;-0,60)$} & \multirow{2}{*}{$9,000 * *$} \\
\hline Subescala 4 da CAMI-BR - Ideologia Comunitária de Saúde Mental & $3,13(0,54)$ & & \\
\hline Subescala 2 da CAMI-BR - Restrição Social & $2,79(0,50)$ & \multirow{2}{*}{$(-0,64 ;-0,41)$} & \multirow{2}{*}{$2,000^{* *}$} \\
\hline Subescala 3 da CAMI-BR - Benevolência & $3,31(0,40)$ & & \\
\hline Subescala 2 da CAMI-BR - Restrição Social & $2,79(0,50)$ & \multirow[b]{2}{*}{$(-0,45 ;-0,23)$} & \multirow[b]{2}{*}{$9,000^{* *}$} \\
\hline Subescala 4 da CAMI-BR - Ideologia Comunitária de Saúde Mental & $3,13(0,54)$ & & \\
\hline Subescala 3 da CAMI-BR - Benevolência & $3,31(0,40)$ & \multirow{2}{*}{$(0,06 ; 0,31)$} & \multirow{2}{*}{0,001} \\
\hline Subescala 4 da CAMI-BR - Ideologia Comunitária de Saúde Mental & $3,13(0,54)$ & & \\
\hline
\end{tabular}

*IC $95 \%$ : intervalo de $95 \%$ de confiança referente à diferença entre as médias; ${ }^{*} p<0,001$.

\subsection{Análise dos escores por itens da CAMI -BR}

Nas Tabelas 2 e 3, estão descritos os dados dos itens específicos da escala, por meio das médias dos postos de Friedman e das porcentagens de respostas aos itens. Com relação às porcentagens, os valores da coluna 0 e 1 indicam atitudes negativas, os valores da coluna 2 indicam atitudes neutras e os valores dispostos na coluna 3 e 4 indicam atitudes positivas.

A Tabela 2 apresenta os resultados referentes às subescalas de Autoritarismo e de Restrição Social. No que se refere à primeira, a afirmação de que "a melhor forma de controlar os doentes mentais é trancá-los" (item 2; média dos postos $=8,09$ ) foi, significativamente, a que mais contribuiu para as atitudes dos entrevistados nessa 
subescala $\left(X^{2}=379,394 ; p<0,001\right)$. Com relação à ocorrência de atitudes positivas, a maioria dos sujeitos $(96,4 \%)$ discordou de que "a melhor forma de controlar os doentes mentais é trancá-los" (item 2) e $92,7 \%$ concordaram que "doentes mentais não deveriam ser tratados como indesejáveis" (item 7). No que diz respeito à ocorrência de atitudes negativas, $88,2 \%$ concordaram que "certas características diferenciam os doentes mentais das demais pessoas" (item 3). Por outro lado, dois itens dividiram as opiniões dos entrevistados, apresentando porcentagens semelhantes de respostas indicativas de atitudes positivas e negativas. No que se refere ao primeiro (item 4), 55,4\% discordaram e $43,7 \%$ concordaram com a afirmação de que "a pessoa com sinal de doença mental sempre deve ser hospitalizada". Quanto ao segundo (item 8), 48,2\% dos entrevistados discordaram e $47,3 \%$ concordaram com a afirmação de que "deve ser dada menos importância em proteger a sociedade dos doentes mentais".

Com relação à subescala de Restrição Social, a afirmação de que "doentes mentais deveriam ser isolados da comunidade" (item 12; média dos postos $=7,69)$ foi, significativamente, a que mais contribuiu para as atitudes dos sujeitos nessa subescala $\left(X^{2}=\right.$ $368,914 ; p<0,001)$. Com relação à ocorrência de atitudes positivas, a maioria $(98,2 \%)$ dos sujeitos discordou que os "doentes mentais deveriam ser isolados da comunidade" (item 12); $94,6 \%$ concordaram que "ninguém tem o direito de excluir os doentes mentais da vizinhança" (item 18) e 89,1\% discordaram de que "não gostariam de ser vizinhos de porta de um doente mental" (item 14). Quanto à ocorrência de atitudes negativas, 76,4\% discordaram que "a maioria das mulheres com histórico em hospital psiquiátrico pode ser confiável como babá" (item 20) (Tabela 2). 
Joanna Gonçalves de Andrade Tostes, Marina Bandeira, Marcos Santos de Oliveira

Tabela 2

Resultados de cada item das subescalas de Autoritarismo (1-10) e Restrição social (11-20), em termos de médias dos postos de Friedman e porcentagens de respostas aos itens

\begin{tabular}{|c|c|c|c|c|}
\hline \multirow{2}{*}{ Resumo dos itens } & \multirow{2}{*}{$\begin{array}{c}\text { Médias } \\
\text { dos } \\
\text { postos }\end{array}$} & \multicolumn{3}{|c|}{ Porcentagens ( $\%)$} \\
\hline & & 0 e $1^{*}$ & $2 *$ & $3 \mathrm{e} 4^{*}$ \\
\hline \multicolumn{5}{|l|}{ AUTORITARISMO } \\
\hline $\begin{array}{l}\text { 1. Uma das principais causas da doença mental (DM) é a falta de } \\
\text { autodisciplina e autocontrole }\end{array}$ & 5,32 & $36,4 \%$ & $0,9 \%$ & $62,7 \%$ \\
\hline 2. Melhor forma de controlar doentes mentais é trancá-los & $8,09 * *$ & $3,6 \%$ & - & $96,4 \%$ \\
\hline 3. Certas caracteristicas diferenciam doentes mentais das demais pessoas & 2,80 & $88,2 \%$ & $3,6 \%$ & $8,2 \%$ \\
\hline 4. Pessoa com sinal de DM sempre deve ser hospitalizada & 4,90 & $43,7 \%$ & $0,9 \%$ & $55,4 \%$ \\
\hline 5. Doentes mentais e crianças precisam do mesmo controle e disciplina & 4,07 & $59,1 \%$ & $3,6 \%$ & $37,3 \%$ \\
\hline 6. DM é uma doença como outra qualquer & 4,13 & $59,1 \%$ & - & $40,9 \%$ \\
\hline 7. Doentes mentais não deveriam ser tratados como indesejáveis & 7,71 & $7,3 \%$ & - & $92,7 \%$ \\
\hline $\begin{array}{l}\text { 8. Deveria ser dada menosimportância emproteger a sociedade dos } \\
\text { doentes mentais }\end{array}$ & 4,68 & $48,2 \%$ & $4,5 \%$ & $47,3 \%$ \\
\hline 9. Hospitais psiquiátricos são modelos ultrapassados de tratamento & 6,35 & $22,7 \%$ & $2,7 \%$ & $74,6 \%$ \\
\hline 10. Qualquer um pode se tomarum doente mental & 6,95 & $10 \%$ & $1,8 \%$ & $88,2 \%$ \\
\hline \multicolumn{5}{|l|}{ RESTRIÇÃO SOCIAL } \\
\hline 11.Nenhuma responsabilidade deveria ser $\mathrm{dada}$ aos doentes mentais & 4,61 & $26,4 \%$ & $0,9 \%$ & $72,7 \%$ \\
\hline 12. Doentes mentais deveriam ser isolados da comunidade & $7,69 * * *$ & $1,8 \%$ & - & $98,2 \%$ \\
\hline 13. Uma mulher é tola em se casar com um doente mental & 4,66 & $26,4 \%$ & $5,5 \%$ & $68,1 \%$ \\
\hline 14.Não gostaria de ser vizinho de porta de um doente mental & 6,73 & $7,3 \%$ & $3,6 \%$ & $89,1 \%$ \\
\hline 15. Pessoa com histónico de DM não deveria assumir emprego público & 5,39 & $13,6 \%$ & $0,9 \%$ & $85,5 \%$ \\
\hline 16. Doentes mentais não deveriam ser privados dos direitos individuais & 6,04 & $10,9 \%$ & $0,9 \%$ & $88,2 \%$ \\
\hline 17. Doentes mentais devem ser encorajados a responsabilidades normais & 5,22 & $20,0 \%$ & $0,9 \%$ & $79,1 \%$ \\
\hline 18. Ninguém temo direito de excluir os doentes mentais da vizinhança & 7,08 & $4,5 \%$ & $0,9 \%$ & $94,6 \%$ \\
\hline 19. Doentes mentais oferecemmenos perigo do que as pessoas pensam & 5,55 & $17,4 \%$ & $3,7 \%$ & $78,9 \%$ \\
\hline $\begin{array}{l}\text { 20. Maioria das mulheres com histórico em hospitais psiquiátricos } \\
\text { pode ser confiável como babá }\end{array}$ & 2,03 & $76,4 \%$ & $5,5 \%$ & $18,1 \%$ \\
\hline
\end{tabular}

* 0 e 1 = atitudes negativas; 2 = atitudes neutras; 3 e 4 = atitudes positivas

**Subescala 1 - Autoritarismo (1-10): Chi,Square $=379,394 ; \mathrm{df}=9 ; p<0,001$

***Subescala 2 - Restrição Social (11-20): Chi-Square $=368.914 ; \mathrm{df}=9 ; p<0,001$

A Tabela 3 apresenta os resultados referentes às subescalas de Benevolência e de Ideologia Comunitária de Saúde Mental. Na primeira, os resultados das médias dos postos de Friedman mostraram que a afirmação "doentes mentais não merecem nossa simpatia" (item 26; média dos postos $=6,59$ ) foi, significativamente, a mais relevante para as atitudes dos sujeitos nessa subescala $\left(X^{2}=\right.$ $86,844 ; p<0,001)$. Todos os itens desta subescala mostraram que a maioria dos sujeitos (superiores a $80 \%$ ) apresentava atitudes positivas, tais como: $100 \%$ discordaram que "doentes mentais não merecem nossa simpatia" (item 26); 98,2\% discordaram que 
"aumentar gastos com saúde mental é desperdício" (item 28) e $99,1 \%$ concordaram que "precisamos ser mais tolerantes com os doentes mentais" (item 23).

Na subescala de Ideologia Comunitária de Saúde mental, o teste de Friedman mostrou que a afirmação de que "é assustador pensar em pessoas com doença mental morando em bairros residenciais" (item 39; média dos postos $=6,20$ ) foi, significativamente, a mais importante para as atitudes dos entrevistados nessa subescala $\left(X^{2}=\right.$ $42,067 ; \mathrm{p}<0,001)$. Os resultados indicaram atitudes positivas da maioria dos entrevistados, sendo que $97,3 \%$ dos sujeitos concordaram que "a assistência aos doentes mentais deveria ser oferecida na comunidade" (item 33); 94,5\% discordaram que "os serviços de saúde mental deveriam ser mantidos longe de bairros residenciais" e 94,6\% discordaram que "é assustador pensar em pessoas com doença mental morando em bairros residenciais" (item 39) (Tabela 3). 
Joanna Gonçalves de Andrade Tostes, Marina Bandeira, Marcos Santos de Oliveira

Tabela 3

Resultados de cada item das subescalas de Benevolência (21-30) e Ideologia Comunitária de Saúde Mental (31-40), em termos de médias dos postos de Friedman e porcentagens de respostas aos itens

\begin{tabular}{|c|c|c|c|c|}
\hline \multirow{2}{*}{ Resumo dos itens } & \multirow{2}{*}{$\begin{array}{c}\text { Médias } \\
\text { dos } \\
\text { postos }\end{array}$} & \multicolumn{3}{|c|}{ Porcentagens (\%) } \\
\hline & & $0 \mathrm{e} 1^{*}$ & $2 *$ & 3 e $4^{*}$ \\
\hline \multicolumn{5}{|l|}{ BENEVOLENCIA } \\
\hline 21. Doentes mentais foram por muito tempo ridicularizados & 5,78 & $6,4 \%$ & - & $93,6 \%$ \\
\hline $\begin{array}{l}\text { 22. Deveriam ser gastos mais recursos públicos no tratamento de doentes } \\
\text { mentais }\end{array}$ & 5,76 & $3,6 \%$ & $2,7 \%$ & $93,7 \%$ \\
\hline 23. Precisamos ser mais tolerantes com doentes mentais & 6,00 & $0,9 \%$ & - & $99,1 \%$ \\
\hline $\begin{array}{l}\text { 24. Hospitais psiquiátricos parecem mais prisões do que locais para } \\
\text { tratamento }\end{array}$ & 5,27 & $10,9 \%$ & $5,5 \%$ & $83,6 \%$ \\
\hline $\begin{array}{l}\text { 25. Sociedade é responsável por dar o melhor tratamento a os doentes } \\
\text { mentais }\end{array}$ & 5,21 & $7,3 \%$ & $\cdot$ & $92,7 \%$ \\
\hline 26. Doentes mentais não merecem nossa simpatia & $6,59 * *$ & - & - & $100 \%$ \\
\hline 27. Doentes mentais sã $Q$ um peso para a sociedade & 5,53 & $4,5 \%$ & - & $95,5 \%$ \\
\hline 28. Aumentar gastos com saúde mental é desperdicio & 5,96 & $1,8 \%$ & - & $98,2 \%$ \\
\hline 29. Existem serviços de saúde mental suficientes para os doentes mentais & 4,46 & $9,1 \%$ & $2,7 \%$ & $88,2 \%$ \\
\hline 30. É melhor evitar alguém com doença mental(DM) & 4,44 & $12,7 \%$ & $0,9 \%$ & $86,4 \%$ \\
\hline \multicolumn{5}{|l|}{ IDEOLOGIA COMUNITÁRIA DE SAÚDE MENTAL } \\
\hline 31. Todos deveriam aceitar serviços de saúde mentalna vizinhança & 4,90 & $10,0 \%$ & $4,5 \%$ & $85,5 \%$ \\
\hline 32. Melhor terapia para doentes mentais é viver na comunidade & 5,84 & $6,4 \%$ & $1,8 \%$ & $91,8 \%$ \\
\hline $\begin{array}{l}\text { 33. A assistência aos doentes mentais deveria ser oferecida na } \\
\text { comunidade }\end{array}$ & 5,64 & $1,8 \%$ & $0,9 \%$ & $97,3 \%$ \\
\hline $\begin{array}{l}\text { 34. Serviços de saúde mental em bairro residencial não colocam em } \\
\text { perigo os moradores }\end{array}$ & 5,64 & $9,1 \%$ & $1,8 \%$ & $89,1 \%$ \\
\hline $\begin{array}{l}\text { 35. Mora dores não têmo que temer de pessoas que vêm receber } \\
\text { tratamento na vizinhança }\end{array}$ & 5,05 & $10,0 \%$ & $0,9 \%$ & $89,1 \%$ \\
\hline $\begin{array}{l}\text { 36. Serviços de saúde mental deveriam ser mantidos longe de bairros } \\
\text { residenciais }\end{array}$ & 5,70 & $5,5 \%$ & - & $94,5 \%$ \\
\hline $\begin{array}{l}\text { 37. Moradores têm boas razões para resistir à implantação de serviços de } \\
\text { saúde mentalna vizinhança }\end{array}$ & 5,22 & $8,2 \%$ & $0,9 \%$ & $90,9 \%$ \\
\hline $\begin{array}{l}\text { 38. Morar em áreas residenciais pode ser uma boa terapia para doentes } \\
\text { mentais, mas o risco para os mora dores é grande }\end{array}$ & 4,93 & $13,6 \%$ & $1,8 \%$ & $84,6 \%$ \\
\hline $\begin{array}{l}\text { 39. É assusta dor pensar em pessoas com DM morando em bairros } \\
\text { residenciais }\end{array}$ & $6,20 * * *$ & $4,5 \%$ & $0,9 \%$ & $94,6 \%$ \\
\hline 40. Serviços de saúde mental em área residencial desvalonizam o bairro & 5,87 & $4,5 \%$ & $2,7 \%$ & $92,8 \%$ \\
\hline
\end{tabular}

* 0 e 1 = atitudes negativas; 2 = atitudes neutras; 3 e 4 = atitudes positivas

**Subescala 3 - Benevolência (21-30): Chi-Square $=86,844 ; \mathrm{df}=9 ; p<0,001$

***:Subescala 4 - Ideologia Comunitána de Saúde Mental (31-40): Chis Sauqre $=42,067 ; \mathrm{df}=9 ; p<0,001$

\section{Discussão}

Os resultados deste estudo permitiram conhecer as atitudes dos moradores da vizinhança dos Serviços Residenciais Terapêuticos (SRTs) em relação aos pacientes psiquiátricos e aos serviços de 
saúde mental. Além disso, possibilitaram a identificação dos domínios e dos itens nos quais essas atitudes se mostraram mais positivas ou mais negativas. O escore global da CAMI-BR apontou para atitudes entre neutras e positivas, semelhante ao resultado encontrado por Abelha et al. (2015). Houve também correspondência nos resultados destes dois estudos para as subescalas Autoritarismo (atitudes do neutro ao positivo) e Ideologia Comunitária de Saúde Mental (atitudes positivas). Por outro lado, houve diferenças na subescala de Restrição Social, que no presente estudo indicou atitudes do neutro ao positivo e no estudo de Abelha et al. (2015) atitudes positivas, enquanto que o resultado inverso foi observado para a subescala de Benevolência.

No estudo de Wahl (1993), que avaliou os vizinhos e não vizinhos de serviços residenciais para pacientes psiquiátricos, houve diferença com o presente estudo na única subescala da CAMI que o autor utilizou, a de Ideologia Comunitária de Saúde Mental. As atitudes variaram do neutro ao negativo para ambos os grupos de sujeitos, diferentemente do presente estudo e da pesquisa de Abelha et al. (2015), nos quais as atitudes foram positivas para esta subescala.

O estudo de Wolff et al. (1996), ao avaliar vizinhos desses serviços, apresentou seus resultados em termos de porcentagens de respostas aos itens específicos da CAMI. Com relação às atitudes negativas, $14 \%$ dos entrevistados discordaram da afirmação de que "serviços de saúde mental em bairro residencial não colocam em perigo os moradores" (item 34), enquanto no presente estudo isso ocorreu somente para 9,1\%. Na pesquisa de Wolff et al. (1996), 15\% das pessoas concordaram com a afirmação de que "moradores têm boas razões para resistir à implantação de serviços de saúde mental na vizinhança" (item 37), comparativamente a apenas $8,2 \%$ na presente pesquisa. Em relação à frase "é assustador pensar em pessoas com doença mental morando em bairros residenciais" (item 39), a porcentagem de acordo no estudo de Wolff et al. (1996) foi de $15 \%$ e no presente estudo foi de somente $4,5 \%$. Estes dados indicam atitudes mais negativas dos sujeitos da pesquisa de Wolff et al. (1996), comparativamente ao presente estudo, em três itens da escala. Resultado oposto ocorreu para o item 5 . No presente estudo, a maioria dos sujeitos $(59,1 \%)$ concordou com a afirmação de que "doentes mentais e crianças precisam do mesmo controle e disciplina" (item 5), enquanto no estudo de Wolff et al. (1996) isso ocorreu para $20 \%$ dos entrevistados. No que diz respeito às atitudes positivas, houve uma concordância marcante entre o estudo de Wolff et al. (1996) e o presente estudo. Ambos encontraram alta porcentagem de concordância com o item "a sociedade é responsável por dar o melhor tratamento aos doentes mentais" (item 25), respectivamente, $95 \%$ e $92,7 \%$. No entanto, de forma geral, Wolff et 
al. (1996) indicaram que as atitudes dos vizinhos se mostraram predominantemente negativas.

Quanto aos demais estudos realizados com vizinhos das residências para pacientes psiquiátricos, a maioria aplicou questionários não validados elaborados pelos próprios autores, 0 que limita parcialmente a comparação dos resultados. De modo semelhante ao presente estudo, Aubry et al. (1995) encontraram atitudes positivas. No estudo de Arens (1993), a maioria dos sujeitos relatou que os pacientes psiquiátricos eram bons vizinhos e que não perceberam um impacto negativo no valor das propriedades da região. No presente estudo, o resultado de um dos itens da CAMI-BR apresentou semelhança com estes últimos dados e também com os resultados da pesquisa de Rabkin et al. (1984), pois a grande maioria discordou de que "serviços de saúde mental em área residencial desvalorizam o bairro" (item 40). Porém, resultado distinto foi observado por Cook (1997) que, embora tenha encontrado vizinhos com atitudes predominantemente positivas, ainda assim apresentaram preocupações a respeito do efeito negativo da presença das residências sobre o preço dos imóveis na redondeza e na segurança dos outros moradores.

Nos estudos de Zippay e Lee (2008) e Rabkin et al. (1984) também foram observados resultados equivalentes aos do presente estudo, mostrando que a maioria dos vizinhos não apresentou atitudes negativas direcionadas aos pacientes psiquiátricos ou ao estabelecimento dos serviços de saúde mental na vizinhança. Outro item da CAMI-BR que mostrou resultado semelhante ao encontrado no estudo de Rabkin et al. (1984) se refere à frase "morar em áreas residenciais pode ser uma boa terapia para doentes mentais, mas 0 risco para os moradores é grande" (item 38), sobre a qual a maioria dos entrevistados de ambos os estudos discordou.

Contrariamente aos resultados encontrados no presente trabalho e na maioria dos estudos anteriormente descritos, Reda (1995) constatou a predominância de atitudes negativas, vinculando os pacientes psiquiátricos à violência, agressão física e comportamento bizarro, dentre outros aspectos pejorativos. Além disso, os vizinhos declararam que, do mesmo modo que doenças físicas são tratadas em hospitais gerais, doenças mentais devem ser tratadas em hospitais psiquiátricos. Em concordância com esses resultados, 0 estudo de Trute e Loewen (1978), que utilizou a Escala de Rejeição Social, observou atitudes negativas no escore total e na subescala de Rejeição nas Relações Sociais. Na pesquisa de Brunton (1997), que entrevistou grupos de vizinhos e não vizinhos, seis meses antes e seis meses após a mudança dos pacientes para a vizinhança, não houve diferenças consideráveis nas atitudes dos sujeitos de ambos os grupos, de forma geral. Porém, o autor destacou que em um dos itens do questionário utilizado as atitudes se tornaram mais 
negativas, pois grande porcentagem de pessoas dos dois grupos concordou com a afirmação de que "todos os pacientes psiquiátricos devem ser tratados em hospitais". Esse resultado se aproxima do obtido no presente trabalho, no qual quase a metade dos entrevistados concordou com o item da escala CAMI-BR de que "a pessoa com sinal de doença mental sempre deve ser hospitalizada" (item 4).

Pode-se concluir que os resultados do presente estudo apontam, em geral, para uma relativa aceitação dos pacientes e dos SRTs pela vizinhança. A aceitabilidade das pessoas da comunidade em relação aos serviços fornecidos constitui um indicador de qualidade do cuidado em saúde mental, conforme proposto por Donabedian (1990) e recomendado pela OMS (2001). No entanto, na análise dos domínios e dos itens da escala, as atitudes foram mais positivas para alguns e mais negativas para outros. Na subescala de Benevolência, as atitudes foram mais positivas e na de Autoritarismo foram mais negativas. Por exemplo, mais da metade da amostra concordou que pacientes e crianças necessitam do mesmo controle e disciplina e discordou que o transtorno psiquiátrico é uma doença como outra qualquer. Além disso, quase a metade concordou que as pessoas com sinais de transtornos psiquiátricos devem ser hospitalizadas para receber tratamento. Estes resultados mostram certa ambivalência ou relatividade das atitudes das pessoas e apontam para a necessidade de fornecer mais informações à população sobre os transtornos psiquiátricos.

Estudos futuros deverão aprofundar o conhecimento sobre as atitudes das pessoas da comunidade a respeito dos pacientes psiquiátricos e dos serviços de saúde mental, permitindo comparações entre os dados obtidos no presente trabalho e dados de outras regiões do país, visando a uma maior generalização dos resultados ou indicando especificidades. Neste estudo, embora a amostragem probabilística permita a generalização estatística dos resultados da amostra para a população-alvo de onde foi retirada, ou seja, para os moradores vizinhos dos SRTs, não é possível generalizar os resultados encontrados para as pessoas que moram distantes desses serviços, 0 que se configura como uma limitação. Portanto, recomenda-se, ainda, a realização de estudos futuros utilizando grupos-controle de não vizinhos de SRTs, para investigar se haverá ou não diferenças em suas atitudes.

É fundamental que sejam desenvolvidas campanhas para informar e sensibilizar a população em geral, sobretudo os vizinhos dos serviços comunitários de saúde mental, não apenas sobre os transtornos psiquiátricos, mas também em relação ao funcionamento desses serviços. Os vizinhos devem se conscientizar do papel relevante que possuem e devem ser orientados sobre formas mais positivas e menos estigmatizantes de lidar com os pacientes 
desinstitucionalizados, de modo a ajudá-los em sua reinserção social na comunidade.

\section{Referências}

Abelha, L., Gonçalves, S., Legay, L., \& Lovisi, G. M. (2014). Atitudes da comunidade em relação aos doentes mentais. In $M$. Bandeira, L. A. Lima, \& S. Barroso. Avaliação de Serviços de Saúde Mental: princípios metodológicos, indicadores de qualidade e instrumentos de medida (pp. 241-259). Rio de Janeiro: Vozes.

Abelha, L., Siqueira, S. R. G., Legay, L., Yang, L., Valencia, E., Sarução, K. R., \& Lovisi, G. M. (2015). Analysis of psychometric properties of the Brazilian Portuguese version of the Community Attitudes towards the Mentally IIl (CAMI-BR). Revista de la Facultad de Ciencias Medicas, 73(3), 211-219.

Amorim, A. K. M. A., \& Dimenstein, M. (2009a). Desinstitucionalização em saúde mental e práticas de cuidado no contexto do serviço residencial terapêutico. Ciência \& Saúde coletiva, 14(1), 195-204. doi: 10.1590/S141381232009000100025

Amorim, A. K. M. A., \& Dimenstein, M. (2009b). Loucura e Cidade: Cenas Biopolíticas e incursões (Des) institucionalizantes. Fractal: Revista de Psicologia, 21(2), 319-336. doi: 10.1590/S1984-02922009000200009.

Arens, D. A. (1993). What do the neighbors think now? Community residences on Long Island, New York. Community Mental Health J ournal, 29, 235-245. doi: 10.1007/BF00778809

Aubry, T. D., Tefft, B., \& Currie, R. F. (1995). Public attitudes and intentions regarding tenants of community mental health residences who are neighbours. Community Mental Health J ournal, 31, 39-52. doi: 10.1007/BF02188979

Bandeira, M. \& Barroso, S. (2005). Sobrecarga das famílias de pacientes psiquiátricos. Jornal Brasileiro de Psiquiatria, 54(1), 34-46.

Barke, A., Nyarko, S., \& Klecha, D. (2011). The stigma of mental illness in Southern Ghana: Attitudes of the urban population and patients' views. Social Psychiatry and Psychiatric Epidemiology, 46, 1191-1202. doi: 10.1007/s00127-010-02903

Brunton, K. (1997). Stigma. Journal of Advanced Nursing, 26, 891898. doi: $10.1046 / j .1365-2648.1997 .00442 . x$

Canhota, C. (2008). Qual a importância do estudo piloto? In E. E. Silva. Investigação passo a passo: Perguntas e respostas para investigação clínica (pp. 69-72). Lisboa: APMCG. 
Contandriopoulos, A. P., Champagne, F., Potvin, L., Denis, J. O., \& Boyle, P. (1994). Saber preparar uma pesquisa. São Paulo: Hucitec Abrasco.

Cook, J. R. (1997). Neighbors' Perceptions of Group Homes. Community Mental Health Journal, 33, 287-299. doi: 10.1023/A:1025043023534

Corrigan, P. W. \& Watson, A. C. (2002). The paradox of self-stigma and mental illness. Clinical Psychology: Science and Practice, 9, 35-53. doi: 10.1093/clipsy.9.1.35

Donabedian, A. (1990). The seven pillars of quality. Archives of Pathology \& Laboratory Medicine, 114(11), 1115-1118.

George, D. \& Mallery, M. (2010). Using SPSS for Windows step by step: A simple guide and reference. Boston: Allyn \& Bacon.

Gonçalves, S., Abelha, L., Legay, L. F., \& Lovisi, G. M. (2008). Equivalência conceitual e semântica da versão em português da escala Community Attitudes Toward Mentally III (CAMI). Caderno de Saúde Coletiva (Rio de Janeiro), 16(4), 749-764.

Griffiths, K. M., Nakane, Y., Christensen, H., Yoshioka, K., Jorm, A. F., \& Nakane, H. (2006). Stigma in response to mental disorders: a comparison of Australia and Japan. BMC Psychiatry, 6, 1-12. doi: 10.1186/1471-244X-6-21

Guillemin, F., Bombardier, C., \& Beaton, D. (1993). Cross-Cultural adaptation of health related quality of life measures: Literature review and proposed guidelines. Journal of Clinical Epidemiology, 46, 1417-1432. doi: 10.1016/08954356(93)90142-N

Liu, J., Yan F., Ma, X., Guo, H. L., Tang, Y. L., Rakofsky, J. J., Wu, X. M., Li, X. Q., Zhu, H., Guo, X. B., Yang, Y., Li, P., Cao, X. D., Li, H. Y., Li, Z. B., Wang, P., \& Xu, Q. Y. (2016). Perceptions of public attitudes towards persons with mental illness in Beijing, China: results from a representative survey. Social Psychiatry and Psychiatric Epidemiology, 51(3), 443-453.

Maclean (1969). Community attitudes to mental illness in Edinburg. British J ournal of Preventive \& Social Medicine, 23, 45-52.

Mari, J. J. (2014). Mental healthcare in Brazil: Modest advances and major challenges. Advances in Psychiatric Treatment, 20, 113115. doi: 10.1192/apt.bp.113.011593

Ministério da Saúde. (2004). Legislação em saúde mental: 1990-2004 (5a. ed.). Brasília, DF: Ministério da Saúde. Recuperado em 1 de agosto, 2013, de http://bvsms.saude.gov.br/bvs/publicacoes/legislacao_saude_ mental_1990_2004_5ed.pdf

Ministério da Saúde. (2010). Relatório Final da IV Conferência Nacional de Saúde Mental - Intersetorial, 27 de junho a 1 de julho de 2010. Brasília: Conselho Nacional de Saúde/Ministério da Saúde. Recuperado em 26 de abril, 2015, de 
http://conselho.saude.gov.br/biblioteca/Relatorios/relatorio_fin al_IVcnsmi_cns.pdf

Ministério da Saúde. (2011). Portaria GM n. 3.090, de 23 de dezembro de 2011. Brasília, DF. Recuperado em 25 de março, 2015, http://bvsms.saude.gov.br/bvs/saudelegis/gm/2011/prt3090_2 3_12_2011_rep.html

Ministério da Saúde. (2015). Saúde Mental em Dados - 12. Informativo eletrônico de dados sobre a Política Nacional de Saúde Mental. SAS/DAPES. Coordenação Geral de Saúde Mental, Álcool e Outras Drogas, 10(12), Brasília, DF. Recuperado em 16 de dezembro de 2015, de www.saude.gov.br/bvs/saudemental

Mirnezami, H. F., Jacobsson, L., \& Edin-Liljegren, A. (2015). Changes in attitudes towards mental disorders and psychiatric treatment 1976-2014 in a Swedish population. Nordic Journal of Psychiatry, 70(1), 38-44. doi: 10.3109/08039488.2015.1046916

Morgado, A. \& Lima, L. A. (1994). Desinstitucionalização: Suas bases e a experiência internacional. Jornal Brasileiro de Psiquiatria (Rio de Janeiro), 43(1), 19-28.

Organização Mundial de Saúde, [OMS]. (2001). Relatório Mundial da Saúde. Saúde mental: nova concepção, nova esperança. Lisboa: Divisão de Saúde Mental da OMS. Recuperado em 27 de maio, 2013, http://www.who.int/whr/2001/en/whr01_djmessage_po.pdf

Organização Mundial de Saúde, [OMS]. (2008). Integração da saúde mental nos cuidados de saúde primários: uma perspectiva global. Lisboa: Divisão de Saúde Mental da OMS. Recuperado em 20 de agosto, 2014, de http://www.who.int/eportuguese/publications/Integracao_saud e_mental_cuidados_primarios.pdf

Rabkin, J. G, Muhlin, G., \& Cohen, P. W. (1984). What the neighbors think: Community attitudes toward local psychiatric facilities. Community Mental Health Journal, 20, 304-12. doi: 10.1007/BF00757079

Reda, S. (1995). Attitudes towards community mental health care of residents in North London. Psychiatric Bulletin, 19, 731-733. doi: $10.1192 / \mathrm{pb} \cdot 19.12 .731$

Resende, M. D. V., Sturion, J. A., \& Pereira, J. C. D. (2004). Tamanho amostral para deteç̧ão de diferenças significativas entre tratamentos. Boletim de Pesquisa Florestal, 49, 109-120.

Selltiz, C., Wrightsman, L. S., \& Cook, S. W. (1987). Métodos de pesquisa nas relações sociais (Vol. 1. Delineamentos de pesquisa). São Paulo: E.P.U. 
Smith, G. R., Manderscheid, R. W., Flynn, L. M., \& Steinwatchs, D. M. (1997). Principles for assessment of patient outcomes in mental health care. Psychiatric Services, 48(8), 1033-1036.

Snedecor, G. W. \& Cochran, W. G. (1971). Métodos estatísticos. México: Editora Continental.

Taylor, S. M. \& Dear, M. J. (1981). Scaling community attitudes toward the mentally Ill. Schizophrenia Bulletin, 7, 225-240. doi: $10.1093 / \mathrm{schbul} / 7.2 .225$

Thornicroft, G. \& Tansella, M. (2010). Saúde Mental da população e cuidado comunitário. In G. Thornicroft \& M. Tansella. Boas práticas em Saúde Mental Comunitária (pp. 6-68). Barueri: Manole.

Trute, B. \& Loewen, A. (1978). Public attitude toward the mentally ill as a function of prior personal experience. Social Psychiatry, 13, 79-84. doi: 10.1007/BF00583864

Wahl, O. F. (1993). Community impact of group homes for mentally ill adults. Community Mental Health Journal, 29, 247-259, doi: 10.1007/BF00778810

Wolff, G., Pathare, S., Craig, T., \& Leff, J. (1996). Community attitudes to mental illness. British Journal of Psychiatry, 168, 183-190. doi: 10.1192/bjp.168.2.183

World Health Organization. [WHO]. (2013). Mental health action plan 2013-2020. WHO Library Cataloguing-in-Publication Data. Geneva: WHO. Recuperado em 13 de novembro, 2015, de http://apps.who.int/iris/bitstream/10665/89966/1/9789241506 021_eng.pdf?ua $=1$

Zippay, A. \& Lee, S. K. (2008). Neighbors' perceptions of communitybased psychiatric housing. Social Service Review, 82(3), 395417.

\footnotetext{
Endereço para correspondência

J oanna Gonçalves de Andrade Tostes

Universidade Federal de São João del-Rei - UFS]

Programa de Pós-Graduação Stricto Sensu em Psicologia

Laboratório de Pesquisa em Saúde Mental (LAPSAM) - Campus Dom Bosco

Praça Dom Helvécio, 74, Fábricas, CEP 36301-160, São João del-Rei - MG, Brasil

Endereço eletrônico: joanna@tostes.org

Marina Bandeira

Universidade Federal de São João del-Rei - UFSJ

Departamento de Psicologia

Laboratório de Pesquisa em Saúde Mental (LAPSAM) - Campus Dom Bosco

Praça Dom Helvécio, 74, Fábricas, CEP 36301-160, São João del-Rei - MG, Brasil

Endereço eletrônico: bandeira@ufsj.edu.br

Marcos Santos de Oliveira

Universidade Federal de São João del-Rei - UFS]

Departamento de Matemática e Estatística - Campus Santo Antônio

Praça Frei Orlando, 170 sl 4.06, Centro, CEP 36307-352, São João del-Rei - MG, Brasil

Endereço eletrônico: mso@ufsj.edu.br
} 
Joanna Gonçalves de Andrade Tostes, Marina Bandeira, Marcos Santos de Oliveira

Recebido em: 16/06/2016

Reformulado em: 02/08/2017

Aceito em: 02/08/2017

\section{Notas}

* Mestra em Psicologia, com ênfase em Saúde Mental, pelo Programa de PósGraduação Stricto Sensu em Psicologia da UFSJ.

** PhD em Psicologia pela Université de Montréal e pós-doutorado pelo Centre de Recherche Fernand-Seguin e pelo Psychosocial Recherche Center. Professora Titular do Departamento de Psicologia da UFSJ.

*** Doutor em Estatística e Experimentação Agropecuária pela Universidade Federal de Lavras. Professor Associado I do Departamento de Matemática e Estatística da UFSJ.

Financiamento: Coordenação de Aperfeiçoamento de Pessoal de Nível Superior (CAPES).

Este artigo de revista Estudos e Pesquisas em Psicologia é licenciado sob uma Licença Creative Commons Atribuição-Não Comercial 3.0 Não Adaptada. 